\title{
Testing the assumptions and interpreting the results of the Rasch model using log-linear procedures in SPSS
}

\author{
ELISABETH TENVERGERT \\ University Hospital Groningen, Groningen, The Netherlands \\ MICHAEL GILLESPIE \\ University of Alberta, Edmonton, Alberta, Canada \\ and \\ JOHANNES KINGMA \\ University Hospital Groningen, Groningen, The Netherlands
}

\begin{abstract}
This paper shows how to use the log-linear subroutine of SPSS to fit the Rasch model. It also shows how to fit less restrictive models obtained by relaxing specific assumptions of the Rasch model. Conditional maximum likelihood estimation was achieved by including dummy variables for the total scores as covariates in the models. This approach greatly simplifies the specification of the Rasch models. We illustrate these procedures in an analysis of four items selected from the Reiss Premarital Sexual Permissiveness Scale. We found that a modified version of the Rasch model with item dependencies fits the data significantly better than the simple Rasch model. We also found that the item difficulties are the same for men and women, but that the item dependencies are significantly greater for men. Apart from any substantive issues these results raise, the value of this exercise lies in its demonstration of how researchers can use the procedures of popular, accessible software packages to study an increasingly important set of measurement models.
\end{abstract}

Rasch measurement models (Rasch, 1960) have been the focus of much recent work in psychology, education, and sociology (Andrich, 1982, 1985, 1988; Duncan, 1984a, 1984b). The Rasch model belongs to the family of latent trait models and has frequently been recommended as an alternative model to correlation-based methods in testing the unidimensionality of a set of dichotomous iterns (Andrich, 1988; Duncan, 1984b; Hulin, Drasgow, \& Parsons, 1983).

Although the models were specified independently, the Rasch model represents a stochastic version of the earlier Guttman scaling model (Andrich, 1985; Duncan, 1984b; Mokken, 1971). One of the fundamental features of Guttman scaling is that responses to individual items can be uniquely determined from the total score when the items are scalable. A difficulty with Guttman scaling is that it is a deterministic model; it makes no allowance for mea-

This research was supported by University of Utah Research Grant 215173, by Grant A 1235 from the Natural Sciences and Engineering Research Council of Canada, and by Grant H56-314 from the Netherlands Organization for the Advancement of Pure Scientific Research (Z.W.O.). We gratefully acknowledge several anonymous reviewers for helpful comments on earlier drafts of this manuscript. M.G. is in the Department of Sociology at Alberta; J.K. is in the Department of Traumatology at Groningen. Requests for reprints should be sent to E. TenVergert, Thorax Center, University Hospital Groningen, Oostersingel 59, 9713 EZ Groningen, The Netherlands (e-mail: tenv@rugr86.rug.nl). surement error. Since measurement error is ubiquitous in all measures (particularly in measures of attitude), Guttman and others developed ad hoc procedures for deciding whether or not a set of items was scalable and, if so, how to score respondents whose response pattern would suggest one of the scale types. The advantage of the Rasch model over these ad hoc methods is that it builds the possibility of measurement error into the model by positing a probabilistic relation between the values of the latent variable and the probability of a positive response to the item. In particular, the Rasch model specifies a logistic function for the item trace line in place of the Guttman step function in which the probability of response is either one or zero.

There are at least two attractive properties of the Rasch approach that argue for its application to measurement in the social sciences. First, as a latent-trait model, it is consistent with most conceptions of the latent variable as continuous (Duncan, 1984b). Second, the Rasch model poses the question of unidimensionality in terms of the properties of local independence and specific objectivity. Local independence means that responses to the items will be (conditionally) independent, given the respondent's location on the latent variable being measured (Andersen, 1988). The conceptual framework for the property of specific objectivity is closely related to Thurstone's criteria for invariance and relevance (Andrich, 1978) and has a 
special relationship to fundamental measurement in physics (Andrich, 1988; Duncan, 1984a).

The specific objectivity of the Rasch model derives from the fact that only the so-called difficulty parameter varies from item to item. The model specifies the same discrimination parameter (or slope) for each item, so that, like the step functions of the Guttman model, the item trace lines parallel one another (Wright \& Stone, 1979). The two meanings of specific objectivity follow from this invariance: First, if a set of items fits the Rasch model for a given population, the difficulty parameter of the item can be estimated from any sample drawn from the population, regardless of how the sample is constituted. In particular, the estimation of the difficulty parameters for a set of items is unaffected by the distribution of the cases on the latent variable being measured. Second, it allows the researcher to use the total scale score for a particular subject as a sufficient statistic for estimating the position of that case on the latent variable. An implication of this second property is that if a population of items fits the Rasch model, different (but overlapping) samples of items will yield similar estimates of the distances between respondents' positions on the latent variable.

The Rasch model can be stated in a variety of equivalent though apparently different ways (see Langeheine \& Rost, 1988). In the present study, the Rasch model will be presented as a log-linear model (Andersen, 1982; Clogg, 1988; Duncan 1984a; Kelderman, 1984; Mellenberg \& Vijn, 1981). Contingency table methods using loglinear models are applied mostly to sociological research data, whereas in psychological and educational studies, researchers are more familiar with latent trait models. However, when Rasch's model is represented as a loglinear model for contingency tables, it can be tested and estimated by using the log-linear procedure in SPSS. This program is more widely available to researchers than other programs like PML (Gustaffson, 1979), GLIM (Baker \& Nelder, 1978), LOGIST (Wood, Wingersky, \& Lord, 1976), or FREQ (Haberman, 1979). In addition, Mellenberg and Vijn (1981) and Kelderman (1984) argue that the log-linear specification has several advantages over the usual formulation of the Rasch model in terms of a logistic model. They consider as a main advantage the fact that log-linear Rasch enables the researcher to apply the conditional maximum likelihood (CML), which is, from a theoretical point of view, a more sound estimation procedure than the unconditional maximum likelihood (UML) estimation procedure (Andersen, 1973; Fischer, 1974). In the CML, the number of respondents that have answered the item positively (the item total) is a sufficient statistic for the item parameter, and the number of items positively responded to (the sum score) is a sufficient statistic for the person parameter. In other words, the pattern of positive-negative responses can be ignored, since it does not provide any additional information. In addition, the overall fit of the log-linear Rasch to a set of data can readily be assessed by well-developed statistical procedures, and model tests for less restrictive models can easily be specified. ${ }^{1}$
The purpose of the present study is, first, to demonstrate how the procedure LOG LINEAR in SPSS can be used to fit the Rasch model and, second, to show how the procedure can be employed to test the Rasch model against less restrictive Rasch-type models in which specific assumptions of the model are violated. To illustrate the models, we analyze four items from the Reiss Premarital Sexual Permissiveness Scale (Reiss, 1967).

Two substantive questions motivate the discussion of the models using the four items on premarital sexual permissiveness. The first concerns sex differences in the structure of the scale. If we control for male and female differences in permissiveness, are the response patterns to the items the same for male and female respondents? Although respondents are routinely stratified by sex in testing the specific objectivity of the set of items, this comparison is particularly important for the Reiss items because of sex differences in permissiveness (DeLamater, 1986; DeLamater \& MacCorquodale, 1979; Reiss, 1967). As Kelderman (1984) points out, these cross-group comparisons are part of the test of specific objectivity. The second question concerns the possible effect of a person's response to a given item on his/her response to subsequent items (see, e.g., Duncan \& Stenbeck, 1988; Goldstein, 1980; Kempf, 1974). In order to test for these effects, we relax the assumption of local independence and allow dependencies between adjacent item responses within sex and sum score categories. We will also examine whether these effects differ by sex, should they emerge. Since we discuss the log-linear Rasch model in the context of these data, we begin by presenting the data.

\section{METHOD}

\section{The Data}

To illustrate the log-linear Rasch model, we analyze data on four items selected from the Reiss Premarital Sexual Permissiveness Scale (Reiss, 1967). Reiss developed two scales to measure the respondent's approval or disapproval of premarital sexual activity. One scale measures approval of male behavior; the other measures approval of female behavior. Each scale consists of 12 items that result from the cross-classification of three sexual acts under four different conditions. The sexual acts (in order of their presentation to the respondent) are "kissing," "petting," and "full sexual relations." The conditions are the level of involvement of the actor with his/her sexual partner. The four levels (again in order of presentation) are "engaged to be married," "in love with the partner," " 'feelings of strong affection for the partner," and "no particular feelings of affection for the partner."

In Reiss's version of the scale, the respondent first reports either approval or disapproval of the behavior described by a particular item. Then he/she rates the intensity of the approval or disapproval on a three-point scale. This method of eliciting responses enables the researcher to measure the respondent's permissiveness with respect to each item both in terms of a "natural" dichotomy and in terms of a six-point scale. The dichotomy is natural in the sense that it reflects the respondents' judgments rather than results from a more or less artificial collapsing of categories by the researcher. Although Duncan (1984a) applies the Rasch model to polytomous items, we focus on the binary responses to the items in this paper.

The four items we analyze are the last of the set of 24 presented to the respondent: his/her approval of full sexual relations for a female under the four conditions of involvement. We use the fol- 
Table 1

Parameterization of Four Models of Responses to Four Items From the Reiss Scale of Premarital Sexual Permissiveness ( 0 Approve; 1 = Disapprove)

\begin{tabular}{|c|c|c|c|c|c|c|c|c|}
\hline \multirow[b]{2}{*}{ Cell } & \multicolumn{4}{|c|}{ Item } & \multicolumn{4}{|c|}{ Models } \\
\hline & FSC & FSA & FSL & FSE & 1 & 2 & 3 & 4 \\
\hline 1 & 0 & 0 & 0 & 0 & $\sigma_{\mathbf{0} m} *$ & $\sigma_{0 m^{*}}$ & $\alpha_{j k} \alpha_{k l} \sigma_{0 m} *$ & $\alpha_{j k} \alpha_{j k m} \alpha_{k l} \alpha_{k l m} \sigma_{0 m}{ }^{*}$ \\
\hline 2 & 0 & 0 & 0 & 1 & $\sigma_{1 m}$ & $\alpha_{i} \sigma_{1 m}$ & $\alpha_{l} \alpha_{j k} \sigma_{1 m}$ & $\alpha_{l} \alpha_{j k} \alpha_{j k m} \sigma_{1 m}$ \\
\hline 3 & 0 & 0 & 1 & 0 & $\alpha_{k} \sigma_{1 m}$ & $\sigma_{1 m}$ & $\sigma_{1 m}$ & $\sigma_{1 m}$ \\
\hline 4 & 0 & 0 & 1 & 1 & $\alpha_{k} \sigma_{2 m}$ & $\alpha_{l} \sigma_{2 m}$ & $\alpha_{l} \alpha_{k l} \sigma_{2 m}$ & $\alpha_{l} \alpha_{k l} \alpha_{k l m} \sigma_{2 m}$ \\
\hline 5 & 0 & 1 & 0 & 0 & $\alpha_{j} \sigma_{1 m}$ & $\sigma_{1 m}$ & $\alpha_{k l} \sigma_{1 m}$ & $\alpha_{k l} \alpha_{k l m} \sigma_{1 m}$ \\
\hline 6 & 0 & 1 & 0 & 1 & $\alpha_{j} \sigma_{2 m}$ & $\alpha_{1} \sigma_{2 m}$ & $\alpha_{l} \sigma_{2 m}$ & $\alpha_{l} \sigma_{2 m}$ \\
\hline 7 & 0 & 1 & 1 & 0 & $\alpha_{j} \alpha_{k} \sigma_{2 m}$ & $\sigma_{2 m}$ & $\alpha_{j k} \sigma_{2 m}$ & $\alpha_{j k} \alpha_{j k m} \sigma_{2 m}$ \\
\hline 8 & 0 & 1 & 1 & 1 & $\alpha_{j} \alpha_{k} \sigma_{3 m}$ & $\alpha_{l} \sigma_{3 m}$ & $\alpha_{l} \alpha_{j k} \alpha_{k l} \sigma_{3 m}$ & $\alpha_{l} \alpha_{j k} \alpha_{j k m} \alpha_{k l} \alpha_{k l m} \sigma_{3 m}$ \\
\hline 9 & 1 & 0 & 0 & 0 & $\alpha_{i} \sigma_{1 m}$ & $\alpha_{i} \sigma_{1 m}$ & $\alpha_{i} \alpha_{j k} \alpha_{k l} \sigma_{1 m}$ & $\alpha_{i} \alpha_{j k} \alpha_{j k m} \alpha_{k l} \alpha_{k l m} \sigma_{1 m}$ \\
\hline 10 & 1 & 0 & 0 & 1 & $\alpha_{i} \sigma_{2 m}$ & $\alpha_{i} \alpha_{l} \sigma_{2 m}$ & $\alpha_{i} \alpha_{l} \alpha_{j k} \sigma_{2 m}$ & $\alpha_{i} \alpha_{l} \alpha_{j k} \alpha_{j k m} \sigma_{z m}$ \\
\hline 11 & 1 & 0 & 1 & 0 & $\alpha_{i} \alpha_{k} \sigma_{2 m}$ & $\alpha_{i} \sigma_{2 m}$ & $\alpha_{1} \sigma_{2 m}$ & $\alpha_{i} \sigma_{2 m}$ \\
\hline 12 & 1 & 0 & 1 & 1 & $\alpha_{i} \alpha_{k} \sigma_{3 m}$ & $\alpha_{i} \alpha_{1} \sigma_{3 m}$ & $\alpha_{i} \alpha_{l} \alpha_{k l} \sigma_{3 m}$ & $\alpha_{i} \alpha_{l} \alpha_{k l} \alpha_{k l m} \sigma_{3 m}$ \\
\hline 13 & 1 & 1 & 0 & 0 & $\alpha_{i} \alpha_{j} \sigma_{2 m}$ & $\alpha_{i} \sigma_{2 m}$ & $\alpha_{i} \alpha_{k l} \sigma_{2 m}$ & $\alpha_{j} \alpha_{k l} \alpha_{k l m} \sigma_{2 m}$ \\
\hline 14 & 1 & 1 & 0 & 1 & $\alpha_{i} \alpha_{j} \sigma_{3 m}$ & $\alpha_{i} \alpha_{l} \sigma_{3 m}$ & $\alpha_{i} \alpha_{1} \sigma_{3 m}$ & $\alpha_{i} \alpha_{j} \sigma_{3 m}$ \\
\hline 15 & 1 & 1 & 1 & 0 & $\alpha_{i} \alpha_{j} \alpha_{k} \sigma_{3 m}$ & $\alpha_{i} \sigma_{3 m}$ & $\alpha_{i} \alpha_{j k} \sigma_{3 m}$ & $\alpha_{i} \alpha_{j k} \alpha_{j k m} \sigma_{3 m}$ \\
\hline 16 & 1 & 1 & 1 & 1 & $\alpha_{i} \alpha_{j} \alpha_{k} \sigma_{4 m}^{*}$ & $\alpha_{i} \alpha_{l} \sigma_{4 m}{ }^{*}$ & $\alpha_{i} \alpha_{l} \alpha_{j k} \alpha_{k l} \sigma_{4 m}{ }^{*}$ & $\alpha_{i} \alpha_{l} \alpha_{j k} \alpha_{j k m} \alpha_{k l} \alpha_{k l m} \sigma_{4 m}{ }^{*}$ \\
\hline
\end{tabular}

Note-FSC, full sexual relations for the female when her relation with the sex partner is more casual; FSA, full sexual relations for the female when she feels strong affection; FSL, full sexual relations for the female when she is in love; FSE, full sexual relations for the female when she is engaged. *Count fitted exactly under the model.

lowing mnemonics when referring to these items: FSE (full sexual relations for the female when engaged), FSL (full sexual relations for the female when she is in love), FSA (full sexual relations for the female when she feels strong affection), and FSC (full sexual relations for the female when her relation with the sex partner is more casual). We choose these particular items because the issue of whether and under what conditions people would approve of a female engaging in full sexual relations prior to marriage seemed to be at the heart of Reiss's research.

The Reiss scale has been administered in numerous settings since its development in the early sixties (DeLamater, 1986; DeLamater \& MacCorquodale, 1979; Reiss, 1967). The data we analyze come from the National Opinion Research Center (NORC) 1963 amalgam survey (SRS-160). This was the first representative national survey in which these items were used, and they form part of the data used by Reiss (1967) in his benchmark study. The sample consists of a multistage probability sample of the adult population (age 21 and over) of the continental United States. Because Reiss finds marked differences between black and white levels of permissiveness, we analyzed just the data on the white respondents $(n=1,352) .^{2}$

\section{The Log-Linear Rasch Model}

The Rasch model for four dichotomous items, $i, j, k, l$, has four item parameters and one person parameter for each respondent. Let $\alpha_{i}, \alpha_{j}, \alpha_{k}, \alpha_{l}$ be the parameters for items $i, j, k$, and $l$, respectively, and let $\xi_{n}$ be the person parameter (or value of the latent trait) for the $n$th respondent. Following Duncan (1984b, 1987; Tjur, 1982), we derive from this model a $\log$-linear parameterization for the four-way cross-classification presented in Table 1 .

The basic postulate of the Rasch model can be stated in terms of the odds on a negative or unfavorable response. Let the odds on a negative response to item $i$ be defined as $\alpha_{i} \xi_{n}$ for the $n$th respondent, and let the odds for the other items be similarly defined. In this expression, $\alpha_{i}$ represents the difficulty of item $i$, and $\xi_{n}$ represents the score of the $n$th respondent on the latent variable. Although some authors define the difficulty parameter in terms of the odds on a positive response, we find that focusing on the negative response is more consistent with the interpretation of the difficulty parameter given in the literature (Andrich, 1988). For example, we would interpret the difficulty parameter $\alpha_{i}$ as follows: If, in com- parison with some other item (say item $l$ ), the $n$th individual agrees with only one item, $\alpha_{i}$ is the odds that the person disagrees with item $i$ rather than with item $l$. Note that if the Rasch model is true, the odds are constant for all individuals. Also note that this interpretation implicitly highlights a feature of parameter estimation and testing the fit of the Rasch model. Cases in which there is either complete agreement or incomplete agreement provide no information; instead, the information is restricted to cases in which persons agree with some items and disagree with others. Given this formulation, the probability of negative (unfavorable) and positive (favorable) responses to the items are:

$\begin{array}{ccc}\text { Item } & \text { Unfavorable } & \text { Favorable } \\ i & \alpha_{i} \xi_{n} /\left(1+\alpha_{i} \xi_{n}\right) & 1 /\left(1+\alpha_{i} \xi_{n}\right) \\ j & \alpha_{j} \xi_{n} /\left(1+\alpha_{j} \xi_{n}\right) & 1 /\left(1+\alpha_{j} \xi_{n}\right) \\ k & \alpha_{k} \xi_{n} /\left(1+\alpha_{k} \xi_{n}\right) & 1 /\left(1+\alpha_{k} \xi_{n}\right) \\ l & \alpha_{i} \xi_{n} /\left(1+\alpha_{i} \xi_{n}\right) & 1 /\left(1+\alpha_{i} \xi_{n}\right)\end{array}$

As previously mentioned, one of the main postulates of the Rasch model is the assumption of local independence; that is, for each individual respondent, the responses to $i, j, k$, and $l$ are independent. As will be shown later, this assumption is sometimes violated, and other, less restrictive models may be specified in order to examine the nature of the violation. The assumption of local independence allows us to write the joint probabilities as the product of the independent probabilities. Thus, for instance, the probability of a negative response on $i, j$, and $k$, and a positive one on $l$ (response pattern $1,1,1,0$ ) is

$$
\frac{\alpha_{i} \xi_{n}}{1+\alpha_{i} \xi_{n}} * \frac{\alpha_{j} \xi_{n}}{1+\alpha_{j} \xi_{n}} * \frac{\alpha_{k} \xi_{n}}{1+\alpha_{k} \xi_{n}} * \frac{1}{1+\alpha_{l} \xi_{n}}
$$

or

$$
\alpha_{i} \alpha_{j} \alpha_{k} \xi_{n}^{3} / \Delta_{n}
$$

where

$$
\Delta_{n}=\left(1+\alpha_{i} \xi_{n}\right)\left(1+\alpha_{j} \xi_{n}\right)\left(1+\alpha_{k} \xi_{n}\right)\left(1+\alpha_{l} \xi_{n}\right) .
$$

We may now write the general formula of the log-linear Rasch model for the four-way contingency table in the following way:

$$
\operatorname{Pr}\left(h_{i} h_{j} h_{k} h_{l} \mid \xi_{n}\right)=\alpha_{i}^{h_{i}} \alpha_{j}^{h_{j}} \alpha_{k}^{h_{k}} \alpha_{l}^{h_{i}} \xi_{n}^{r} / \Delta_{n},
$$


where $h=1$ or 0 , respectively, depending on a positive or negative response to the particular item. Hence the expression $\left(h_{i}, h_{j}\right.$, $h_{k}, h_{l}$ ) reflects merely the possible response patterns of these four items. The exponent $h$ determines whether the item parameters $\alpha_{i}$, $\alpha_{j}, \alpha_{k}$, and $\alpha_{l}$ are or are not included in the product, since $\alpha_{i}^{0}=$ 1 and $\alpha_{i}^{1}=\alpha_{i}$, and so on. Next, $r=h_{i}+h_{j}+h_{k}+h_{i}$, which appears as the power of $\xi_{n}$, is the number of the items answered negatively or unfavorably.

Corresponding to each probability is the expected frequency $F$ of each response pattern in Table 1, under the model, for a sample size $p$ :

$$
F\left(h_{i} h_{j} h_{k} h_{l}\right)=\alpha_{i}^{h_{i}} \alpha_{j}^{h_{j}} \alpha_{k}^{h_{k}} \alpha_{i}^{h_{l}} \sigma_{r m},
$$

where the $\sigma$ parameters or stratum parameters are complex mixtures of item parameters and person parameters-including the respondent's sex (see Duncan, 1984). Note that $r$ in $\sigma_{r}$ refers to the number of negative or unfavorable responses to the four items $(0 \leq r \leq 4)$, and that $m$ refers to the respondent's sex ( $1=$ male, $2=$ female). Thus, in our example, there are $10 \sigma$ parameters. Because these parameters are sample specific, they are typically regarded as holding no substantive interest, in comparison with the item difficulty and person parameters.

Table 1 presents the combination of parameters for each of the 16 response patterns to the four Reiss items for the Rasch and three related models. Following our decision to focus on negative or unfavorable responses, we code the positive response 0 and the negative response 1 . Consistent with Equation 5 , we use the parameters $\alpha_{i}, \alpha_{j}, \alpha_{k}$, and $\alpha_{l}$ to refer to the difficulty parameters for the items FSC, FSA, FSL, and FSE, respectively. The terms $\alpha_{j k}, \alpha_{j k m}$, $\alpha_{k l}$, and $\alpha_{k l m}$ refer to the item dependency parameters that we describe below. We use the terms $\sigma_{r m}$ for the stratum parameters that pertain to the strata obtained by cross-classifying the respondent's total score with his/her sex. These 16 response patterns in Table 1 pertain to men. The parameters for the response patterns of the women will be the same except in Model 4, where the parameters $\alpha_{j k m}$ and $\alpha_{k l m}$ will equal one.

Note that, in all four models, the $\sigma_{r m}$ parameter will be the same for respondents with the same number of negative responses, regardless of which items are disapproved and which are not. For example, the response patterns $(1,1,0,0),(1,0,1,0),(0,1,1,0)$, and $(0,0,1,1)$ all share the same scale parameter $\sigma_{n m}$ : a sum score of two unfavorable or negative responses to the four items. The $m$ subscript means that these parameters (and, hence, the $F$ ) will differ by the respondent's sex, however. Finally, in all four models, we set the stratum parameter $\sigma_{4}$ equal to one (or zero in the logzero specification) for one of the sex categories (women in our example). As a consequence, we can interpret each $\sigma$ parameter as the odds on the total score denoted by the $\sigma$ parameter, relative to a total score of zero (no positive responses to any of the four items). Because the $\sigma$ parameters differ by the respondent's sex, these odds will also differ by sex. Finally, note that because the extreme response patterns of either a positive response or a negative response to all four items uniquely define the $\sigma_{0 \mathrm{~m}}$ and $\sigma_{4 \mathrm{~m}}$ parameters, respectively, the counts for these response patterns will be exactly the same under all four models (or any other Rasch model). Consequently, these extreme response patterns will not figure either in determining the fit of the model to the data or in the estimation of the item parameters.

The first model is the basic Rasch model, in which we set $\alpha_{l}$ equal to 1 (or zero in the log-linear specification). Setting $\alpha_{l}$ equal to 1 arbitrarily locates the origin of the scale at the difficulty of FSE. The second model constrains Model 1 by setting the difficulty parameters for FSA and FSL equal to one another (i.e., $\alpha_{j}=\alpha_{k}$ ). We do this by changing the origin of the scale from the difficulty of FSE to the difficulty parameters of FSA and FSL. The third model modifies Model 2 by relaxing the assumption of local independence for the item pairs (FSA, FSL) and (FSL, FSE). We use the terms $\alpha_{j k}$ and $\alpha_{k l}$, respectively, to denote the conditional dependencies of FSL on FSA and FSE on FSL. Since these items are adjacent, we can interpret these parameters as transfer parameters, which represent the effect of response to one question on the response to subsequent questions (Kempf, 1974). Model 4 modifies Model 3 by allowing these item dependencies to differ between men and women. We use the term $\alpha_{j k m}$ to denote the sex difference in the dependence of FSL and FSA and the term $\alpha_{k l m}$ to denote the sex difference in the dependence of FSE and FSL:

$$
\begin{aligned}
F\left(h_{a} h_{b} h_{c} h_{d}\right)= & \alpha_{i}^{h_{i}} \alpha_{i}^{h_{l}} \alpha_{j k}^{h_{j} h_{k}+\left(1-h_{j}\right)\left(1-h_{k}\right)} \\
& \times \alpha_{j k m}^{h_{m} h_{j} h_{k}+h_{m}\left(1-h_{j}\right)\left(1-h_{k}\right)} \\
& \times \alpha_{k l}^{h_{k} h_{i}+\left(1-h_{k}\right)\left(1-h_{l}\right)} \\
& \times \alpha_{k l m}^{h_{m} h_{k} h_{l}+h_{m}\left(1-h_{k}\right)\left(1-h_{l}\right)} \sigma_{r m} .
\end{aligned}
$$

Equation 6 presents the modifications of the basic Rasch model contained in Model 4 (see Table 1). Note that the exponents of $\alpha_{j k}$ and $\alpha_{k l}$ will be 1 when the responses to the item pairs are consistent $(0,0$, or 1,1$)$ and 0 when the responses are inconsistent $(0,1$, and 1,0 ). If we code males 1 and females 0 , so that the exponent $h$ equal 1 in the case of men and 0 in the case of women, the exponents of $\alpha_{j k m}$ and $\alpha_{k l m}$ will be 1 only for consistent responses to the item pairs and male respondents; they will be zero otherwise.

\section{SPSS Setup for Log-Linear Rasch Models}

Table 2 contains the SPSS setup for testing the goodness of fit and estimating the parameters for four log-linear models. The data, located after the BEGIN DATA command, are in the form of a $2 \times 2 \times 2 \times 2$ contingency table. Note that we use the NORC codes of one and two for "approval" and "disapproval," respectively, in the case of the four items and for "men" and "women," respectively, in the case of the respondent's gender. The reason for this departure from the dummy coding used elsewhere is that the syntax of the CONTRAST keyword in the LOG-LINEAR subroutine of SPSS requires that the value of the reference category be an integer. As we point out below, we use the CONTRAST keyword with approval as the reference category in order to be consistent with the way we have chosen to parameterize the models in the preceding section. Consequently, we had to use the NORC codes rather than dummy coding. Most other parameterization schemes, of course, will be consistent with dummy coding.

The DATA LIST command reads the table with a LIST format: a free field format, with each case beginning on a new record. The DATA LIST command includes the variable FREQ, which refers to the frequency of the cell defined by values of the five variables. We use the WEIGHT command to weight each case or cell by the cell frequency. These features are not necessary when one is inputting raw data, as opposed to data aggregated into a contingency table.

After defining the data, we use the COMPUTE command to create the five-category variable SUM, which represents the number of negative responses to the four items. Since the positive responses (approval) are coded 1 and the negative responses (disapproval) are coded 2, the variable SUM ranges from 4 (approval on all four items) to 8 (disapproval on all four items). Next, we use the RECODE command to convert four of the five scores on the variable SUM to dummy variables in order to estimate the $\sigma$ parameters in Table 1 . For example, we use the dummy variable $S_{3}$, coded 1 for respondents who score 7 on SUM and 0 for all other respondents, to estimate the nuisance parameter $\sigma_{3}$, which corresponds to approval for one item and disapproval for the other three. Note that we do not compute a dummy variable for a SUM score of 8 , disapproval on all four items. By omitting a dummy variable for this category of SUM, we set the value of the nuisance parameter $\sigma_{4}$ equal to one (or a log-linear value of zero). 
Table 2

SPSS $^{\mathrm{x}}$ Control Cards Used to Fit Four Log-Linear Rasch Models

Title 'Log-Linear Rasch for Four Items from the Reiss Premarital'

DATA LIST LIST/FSC, FSA, FSL, FSE, SEX, FREQ

WEIGHT BY FREQ

COMPUTE SUM=FSE+FSL+FSA+FSC

IF (SUM EQ 4) $\mathrm{SO}=1$

IF (SUM NE 4) $\mathrm{SO}=0$

IF (SUM EQ 5) $S I=1$

IF (SUM NE 5) $\mathrm{Sl}=0$

IF (SUM EQ 6) $S 2=1$

IF (SUM NE 6) $S 2=0$

IF (SUM EQ 7) $S 3=1$

IF (SUM NE 7) $\mathrm{S} 3=0$

LOGLINEAR FSC TO FSE $(1,2)$ BY SEX $(1,2)$ WITH SO TO S3

$/$ PRINT=DEFAULT ESTIM

$/$ CONTRAST $($ SEX) $=$ SIMPLE $(2)$

$/$ CONTRAST(FSC) $=$ SIMPLE $(1)$

$/$ CONTRAST(FSA $)=$ SIMPLE $(1)$

$/$ CONTRAST $($ FSL $)=$ SIMPLE $(1)$

DESIGN=FSC,FSA,FSL,S0,S1,S2,S3,S0 BY SEX,S1 BY SEX,S2 BY SEX,S3 BY SEX

$/$ CONTRAST(SEX) $=$ SIMPLE $(2)$

$/$ CONTRAST(FSC) $=$ SIMPLE $(1)$

$/$ CONTRAST(FSE) $=$ SIMPLE $(1)$

DESIGN=FSC,FSE,S0,S1,S2,S3,S0 BY SEX,S1 BY SEX,S2 BY SEX, S3 BY SEX/

$/$ CONTRAST $($ SEX) $=$ SIMPLE $(2)$

$/$ CONTRAST(FSC) $=$ SIMPLE $(1)$

$/$ CONTRAST(FSE) $=$ SIMPLE $(1)$

$/$ CONTRAST(FSA $)=$ SIMPLE $(1)$

$/$ CONTRAST (FSL) $=$ DEVIATION $(1)$

/DESIGN=FSC,FSE,SO,S1,S2,S3,S0 BY SEX,S1 BY SEX,S2 BY SEX,S3 BY SEX, FSL BY FSA, FSE BY FSL

$/$ CONTRAST(SEX) $=$ SIMPLE $(2)$

$/$ CONTRAST(FSC) $=$ SIMPLE $(1)$

$/$ CONTRAST(FSE) $=$ SIMPLE $(1)$

$/$ CONTRAST(FSA) $=$ SIMPLE $(1)$

$/$ CONTRAST(FSL) $=$ DEVIATION $(1)$

/DESIGN=FSC,FSE,SO,S1,S2,S3,S0 BY SEX,S1 BY SEX,S2 BY SEX,S3 BY SEX,

FSL BY FSA, FSE BY FSL, FSL BY FSA BY SEX, FSE BY FSL BY SEX/ BEGIN DATA

1111159

$\begin{array}{llllll}2 & 1 & 1 & 1 & 1 & 39\end{array}$

$\begin{array}{llllll}12 & 1 & 11 & 0\end{array}$

$\begin{array}{llllll}2 & 2 & 1 & 1 & 1 & 17\end{array}$

1121.10

212110

122110

2221129

$\begin{array}{llllll}1 & 1 & 1 & 2 & 1 & 0\end{array}$

211213

$\begin{array}{llllll}1 & 2 & 1 & 2 & 1 & 0\end{array}$

$22121 \quad 1$

$\begin{array}{llll}1 & 1221 & 0\end{array}$

212213

122212

22221474

$\begin{array}{llllll}1 & 1 & 1 & 12 & 18\end{array}$

$\begin{array}{llllll}2 & 1 & 1 & 12 & 9\end{array}$

$\begin{array}{lllll}1 & 2 & 1 & 12 & 1\end{array}$

$\begin{array}{llllll}2 & 2 & 1 & 1 & 2 & 2\end{array}$

$\begin{array}{llllll}1 & 1 & 2 & 1 & 2 & 0\end{array}$

21212 I

122120

222128

111220

$\begin{array}{llllll}2 & 1 & 1 & 2 & 2 & 1\end{array}$

121220

$\begin{array}{lllll}2 & 2 & 1 & 22 & 0\end{array}$

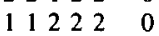

$\begin{array}{llllll}2 & 1 & 2 & 2 & 2 & 1\end{array}$

$\begin{array}{llll}12 & 222 & 0\end{array}$

22222631

END DATA

FINISH 
We use the logit rather than the general form of the log-linear model and place the variable SEX after the keyword BY. This specification takes the distribution of the respondent's sex as given and allows us to test the null hypothesis implied by specific objectivity that the item difficulty parameters are independent of the respondent's sex. We also need to condition on the sum score in order to test another null hypothesis crucial to the Rasch model; that the item difficulty parameters are invariant across the values of the latent variable. We could accomplish this conditioning by also put ting the word SUM after the keyword BY. The problem with this procedure, however, is that it produces a $2 \times 2 \times 2 \times 2 \times 5 \times 2$ table (four dichotomous items, the five-category variable SUM, and the respondent's sex) in which the majority of the 160 cells (128) are empty because they refer to logically impossible combinations of response patterns and SUM (for example, a SUM score of 4 and a response pattern of approval on all four items). Although the SPSS log-linear subroutine allows the user to specify structural zeros with a case weight subcommand (CWEIGHT), this procedure is tedious and undermines the user-friendly features of SPSS. Instead, we achieve conditional maximum likelihood estimation much more simply by including the dummy variables for the categories of SUM, $S_{0}$ through $S_{3}$, as covariates in all the models. We do this by placing the variable names $S_{0}, S_{1}, S_{2}$, and $S_{3}$ after the keyword WITH. Including these variables in each design subcommand allows us to condition on the marginal distribution of the SUM score. In addition, each design subcommand also contains the interaction terms $S_{0}$ by SEX, $S_{1}$ by SEX, $S_{2}$ by SEX, and $S_{3}$ by SEX. These interactions allow us to condition on the joint distribution of the sum score by sex combinations.

The four design subcommands specify the four log-linear models that we describe above. The first model is a Rasch model that assumes local independence of the items with one another and the specific objectivity of invariant item difficulty parameters across the respondent's sex and the sum score. We set the value of the difficulty parameter for the FSE item at one (or a log-linear value of zero) by omitting FSE from the design statement. In effect, this specification arbitrarily sets the origin of the scale equal to the difficulty parameter of FSE. The second model differs from the first in two respects: First, we change the origin of the scale by adding FSE to the design statement and removing FSA and FSL. Second, removal of both items constrains the difficulty parameters for FSA and FSL to be equal. For this reason, the second model is more constrained than the first. The third and fourth models retain the specification that FSA and FSL have the same difficulty but relax the assumption of local independence by allowing for item dependencies between FSL and FSE and between FSL and FSA. The fourth model differs from the third by allowing the magnitudes of the item dependencies to differ for men and women.

Before each design, we include CONTRAST keywords so that the estimate of the difficulty parameter for an item will be consistent with our interpretation of this parameter as the odds on the negative or unfavorable response to the item relative to the odds on a negative response to the reference item (see Table 1). By default, the log-linear subroutine expresses each effect parameter as the deviation from a grand mean. Given our interpretation of the difficulty parameters, on the other hand, we prefer a parameterization that uses the positive response as the reference category. We achieve this result by using a simple, as opposed to deviation, contrast and by specifying the positive category (coded 1) as the reference category. In log-linear terms, this distinction is equivalent to the distinction between analysis of variance and dummy coding. We also express sex differences as a simple contrast between men and women, with women (coded 2) as the reference category. Finally, note that we use the (default) deviation contrast for the FSL item in Models 3 and 4 to make our estimates of the item dependency parameters (more or less) consistent with their expression in Table 1 and Formula 2. Using the deviation contrast means that the item dependency parameters will be estimated as odds rather
Table 3

Percent Approving Full Sexual Relations for an Unmarried Woman for Different Levels of Involvement by Sex of the Respondent

\begin{tabular}{lcc} 
& \multicolumn{2}{c}{ Sex } \\
\cline { 3 - 3 } Level of Involvement & Male* & Female \\
\hline Engaged (FSE) & 23.1 & 5.8 \\
In love (FSL) & 19.0 & 4.6 \\
Affection (FSA) & 16.8 & 4.5 \\
No affection (FSC) & 10.0 & 2.8 \\
\hline
\end{tabular}

$*_{n}=627 . \quad \dagger_{n}=672$.

Table 4

Goodness-of-Fit Statistics for Eight Rasch Models of Responses to Four Items From the Reiss Premarital Permissiveness Scale

\begin{tabular}{cccccc}
\hline \multicolumn{5}{c}{ Goodness-of-Fit Statistics } \\
Model & $d f$ & LR $\chi^{2}$ & $p$ & Pearson $\chi^{2}$ & $p$ \\
\hline 1 & 19 & 39.653 & .004 & 69.940 & .000 \\
2 & 20 & 47.448 & .001 & 68.489 & .000 \\
3 & 18 & 14.101 & .722 & 31.492 & .025 \\
4 & 16 & 9.547 & .889 & 13.858 & .609 \\
$5 \mathrm{a}$ & 15 & 8.162 & .917 & 10.070 & .815 \\
$5 \mathrm{~b}$ & 15 & 9.314 & .861 & 16.264 & .365 \\
$5 \mathrm{c}$ & 15 & 7.138 & .954 & 12.084 & .673 \\
$5 \mathrm{~d}$ & 15 & 9.539 & .848 & 13.992 & .526 \\
\hline
\end{tabular}

Note-See Table 1 for a description of Models 1-4. Models 5a-5d modify Model 4 by allowing for sex differences in the parameters $\alpha_{i}-\alpha_{l}$, respectively.

than as odds ratios. ${ }^{3}$ In its $\log$-linear form, an odds ratio is twice the size of odds (Haberman, 1978).

\section{RESULTS}

The proportion of respondents who approve of full sexual relations for an unmarried woman for the four levels of involvement are given for males and females in Table 3 . We present these results for purposes of description only. Note the low approval of full sexual relations for unmarried women. The percentages for the sample as a whole are $14.8 \%, 11.6 \%, 10.4 \%$, and $6.3 \%$ for the four levels of the relationship between the woman and her partner: engagement, love, affection, and casual. Note also that men are more permissive than women for each level of involvement.

Table 4 contains the goodness-of-fit statistics for the four Rasch models described above (see Tables 1 and 2) plus four additional models that we use to test the null hypothesis that the item difficulty parameter for each of the four items is the same for men and women. The goodness-of-fit statistics are the likelihood-ratio (LR) and Pearson chi-squares, the degrees of freedom, and the $p$ values associated with the chi-square statistics.

Looking at the chi-square values, we see substantial disagreement between the likelihood-ratio and Pearson chisquares, even though these two statistics are asymptotically equivalent. These discrepancies occur because of the large number of cells with very low expected frequencies (see Table 5 below). In such cases, the recommended advice is to choose a model that provides an acceptable fit using both chi-square statistics (Clogg \& Eliason, 1988). Largely on these grounds, we choose Model 4 as 
the one that described the data best. We discuss the basis of our choice in more detail below.

Note, first, that neither of the first two Rasch models achieves acceptable fit, and, in fact, the specification that $\alpha_{j}$ and $\alpha_{k}$ equal one another significantly reduces the fit of Model 2 using LR chi-square (which is more amenable to partitioning than the Pearson chi-square). Nevertheless, we use Model 2 as the baseline model because an analysis of the residuals from Model 1 (not presented) suggests that Model 2 fits the data for women reasonably well; the item-dependency parameters are more pronounced for men than for women. Model 3, which adds the two item-dependency parameters to Model 2, fits significantly better than Model 2 and attains a significant fit using the LR but not the Pearson chi-square. Model 4, which allows the item dependency parameters to differ by sex, achieves an acceptable fit using both chi-squares, although the improvement in fit using the LR chi-square statistic does not attain statistical significance $(p=.109)$. As we shall see, this less than significant improvement occurs because the FSL by FSA by SEX interaction $\left(\alpha_{j k m}\right)$ fails to attain conventional levels of significance. Because its $z$ score (1.773) is sufficiently high, however, we leave it in the model. Finally, note that none of the models that allow the item difficulty parameters to differ by sex, Models 5A-5D, significantly improves on the fit of Model 4 (nor do models that add either the $\alpha_{j}$ or the $\alpha_{l}$ item parameters, which we do not present). Consequently, we choose Model 4 as best representing the data.

Table 5 contains the observed and expected frequencies of responses to the four items for Models 1-4 in Table 4 . Although we present these frequencies primarily to illustrate the estimation of the parameters for the different models, a comparison of the observed frequencies with the expected frequencies may provide some addi- tional insight into the need for item dependencies, particularly in the case of male respondents. Note, first, that most of the women fall into the extreme response categories of either complete disagreement or agreement with all four items. Since estimation of the parameters of the Rasch models is based on the other response categories, the female respondents play a much smaller role than the male respondents in model fit and parameter estimation. Focusing on the male respondents, then, we see high observed frequencies, relative to the expected frequencies under the Rasch model (Model 1), of the following response categories: $(0,1,1,1),(1,0,0,0),(1,0,0,1)$, $(1,0,1,1),(1,1,0,0)$, and $(1,1,1,0)$. These response categories share the common feature of consistent responses to the FSA-FSL or FSL-FSE pairs, or both. We also see low observed frequencies, relative to the expected frequencies under the Rasch model, of the $(1,0,1,0)$ and $(1,1,0,1)$ response categories. Both share the common feature of inconsistent responses to the FSA-FSL and FSL-FSE item pairs. These discrepancies between the observed and expected frequencies occur because of the departure of these item pairs from conditional independence. Note how the expected frequencies for these response categories fit better in the cases of Models 3 and 4 , which allow for these item dependencies.

Table 6 contains the parameter estimates for the four models. Although Models 2-4 set the origin of the scale at the difficulty parameters for FSL and FSA, we recalibrate the parameter estimates by setting the difficulty parameter for FSE equal to zero for all models. This recalibration facilitates comparison of the parameter estimates for the different models. For example, the estimate of $\alpha_{i}$, the item-difficulty parameter for FSC (premarital sex in a casual relationship), stays relatively constant across all four models. Note that we report just

Table 5

Observed and Expected Frequencies of Responses to Four Items From the Reiss Premarital Permissiveness Scale for Men and Women $(0=$ Approve; $1=$ Disapprove)

\begin{tabular}{|c|c|c|c|c|c|c|c|c|c|c|c|c|c|}
\hline \multirow[b]{2}{*}{ FSC } & \multicolumn{8}{|c|}{ Men } & \multicolumn{5}{|c|}{ Women } \\
\hline & FSA & FSL & FSE & $\mathrm{f}$ & F1 & F2 & F3 & F4 & $f$ & F1 & F2 & $\mathrm{F} 3$ & F4 \\
\hline 0 & 0 & 0 & $0^{*}$ & 59.00 & 59.00 & 59.00 & 59.00 & 59.00 & 18.00 & 18.00 & 18.00 & 18.00 & 18.00 \\
\hline 0 & 0 & 0 & 1 & .00 & .11 & .12 & .03 & .01 & 0.00 & .03 & .03 & .01 & .02 \\
\hline 0 & 0 & 1 & 0 & .00 & .53 & .94 & .02 & .00 & 0.00 & .14 & .24 & .00 & .06 \\
\hline 0 & 0 & 1 & I & .00 & .01 & .03 & .09 & .06 & 0.00 & .00 & .01 & .02 & .01 \\
\hline 0 & 1 & 0 & 0 & .00 & 1.44 & .94 & .15 & .05 & 1.00 & .37 & .94 & .04 & .11 \\
\hline 0 & 1 & 0 & 1 & .00 & .04 & .03 & .01 & .00 & 0.00 & .01 & .01 & .00 & .01 \\
\hline 0 & 1 & 1 & 0 & .00 & .20 & .24 & .62 & .45 & 0.00 & .04 & .05 & .12 & .07 \\
\hline 0 & 1 & 1 & 1 & 2.00 & .08 & .09 & 1.51 & 2.11 & 0.00 & .02 & .02 & .39 & .05 \\
\hline 1 & 0 & 0 & 0 & 39.00 & 36.93 & 36.99 & 38.81 & 38.94 & 9.00 & 9.47 & 9.48 & 9.95 & 9.81 \\
\hline 1 & 0 & 0 & 1 & 3.00 & 1.01 & 1.22 & 2.67 & 2.76 & 1.00 & .20 & .24 & .53 & .46 \\
\hline 1 & 0 & 1 & 0 & .00 & 5.02 & 9.24 & 1.70 & .84 & 1.00 & 1.00 & 1.85 & .34 & 1.28 \\
\hline 1 & 0 & 1 & 1 & 3.00 & 2.02 & 3.65 & 4.11 & 3.95 & 1.00 & .52 & .94 & 1.06 & .86 \\
\hline 1 & 1 & 0 & 0 & 17.00 & 13.71 & 9.24 & 14.91 & 15.89 & 2.00 & 2.74 & 1.85 & 2.98 & 2.17 \\
\hline 1 & 1 & 0 & 1 & 1.00 & 5.52 & 3.65 & .47 & .21 & 0.00 & 1.42 & .94 & .12 & .51 \\
\hline 1 & 1 & 1 & 0 & 29.00 & 27.38 & 27.60 & 28.92 & 28.74 & 8.00 & 7.04 & 7.10 & 7.44 & 7.59 \\
\hline 1 & 1 & 1 & $1^{*}$ & 474.00 & 474.00 & 474.00 & 474.00 & 474.00 & 631.00 & 631.00 & 631.00 & 631.00 & 631.00 \\
\hline
\end{tabular}

Note-FSC, full sexual relations for the female when her relation with the sex partner is more casual; FSA, full sexual reations for the female when she feels strong affection; FSL, full sexual relations for the female when she is in love; FSE, full sexual relations for the female when she is engaged; f, observed frequency; F1, F2, F3, and F4, expected frequency for Models 1-4, respectively. ${ }^{*}$ The counts for these cells are fitted exactly in all models. 
Table 6

Parameter Estimates for Four Log-Linear Rasch Models of Four Items From the Reiss Premarital Permissiveness Scale

\begin{tabular}{lccccc}
\hline Margin fit & Parameter & Model 1 & Model 2 & Model 3 & Model 4 \\
\hline FSC & $\alpha_{i}$ & 5.85 & 5.67 & 5.05 & 5.56 \\
FSA & $\alpha_{j}$ & 2.60 & $2.02^{*}$ & $1.84^{*}$ & $1.87^{*}$ \\
FSL & $\alpha_{k}$ & 1.60 & 2.02 & 1.84 & $1.87^{*}$ \\
FSE & $\alpha_{l}$ & $0.00 \dagger$ & 0.00 & 0.00 & 0.00 \\
FSL $\times$ FSA & $\alpha_{j k}$ & 0.00 & 0.00 & 2.29 & 0.84 \\
FSL $\times$ FSA $\times$ SEX & $\alpha_{j k m}$ & 0.00 & 0.00 & 0.00 & 2.22 \\
FSE $\times$ FSL & $\alpha_{k l}$ & 0.00 & 0.00 & 2.17 & 0.52 \\
FSE $\times$ FSL $\times$ SEX & $\alpha_{k l m}$ & 0.00 & 0.00 & 0.00 & 2.45 \\
\hline
\end{tabular}

Note-FSC, full sexual relations for the female when her relation with the sex partner is more casual; FSA, full sexual relations for the female when she feels strong affection; FSL, full sexual relations for the female when she is in love; FSE, full sexual relations for the female when she is engaged. *These parameter estimates are constrained to equal one another. TThe value of 0.00 is fixed rather than estimated.

the item-difficulty and item-dependence parameters, since the "nuisance" parameters $\sigma_{0}-\sigma_{4}$ and their relation to the respondent's sex provide no useful information.

As we have pointed out earlier in this paper, the itemdifficulty parameters represent the log of the odds on a negative response to the item in question, relative to a negative response to the reference item (FSE, premarital sex when the couple is engaged, in the case of Models 1-4), when persons choose only one of the two items. We can gain some insight into this interpretation by using the expected frequencies to calculate some of the parameter estimates. The parameterization of the models in Table 1 provides a guide to this calculation. For example, dividing the expected cell frequency of cell 9 by the expected cell frequency for cell 2 yields an estimate of $\alpha_{i}$, the item difficulty for FSC under Model 1, the Rasch model. The response patterns are $(1,0,0,0)$ and $(0,0$, $0,1)$ for cells 9 and 2 , respectively, and the expected frequencies for Model 1 are 36.93 and .11, respectively (see Table 5). Note that the responses for item FSA and FSL and the sum score are the same for both cells. Thus, the odds of responding negatively to FSC rather than FSE, controlling for the responses to the other items and the sum score, are 335.73 to 1 , or log odds of 5.82. Subject to rounding error in the report of the expected frequencies, three other ratios provide identical estimates of the parameter $\alpha_{i}$ : the expected frequencies in cells 11,13 , and 15 divided by the expected frequencies in cells 4,6 , and 8 .

The addition of item dependencies complicates the estimation of the parameters for Models 3 and 4. Returning to Table 1, we see that we can obtain four estimates of the FSL by FSA item-dependency parameter, $\alpha_{k l}$, fairly simply by dividing the expected frequencies in cells 4 , 5,12 , and 13 by the expected frequencies in cells 6,3 , 14 , and 11, respectively. (Subject to rounding error, these four estimates will equal one another.) Taking the ratio of the expected frequencies in cells 13 and 11 for men in Model 3, for example, we get odds of 8.76 (14.91/1.70) and the log odds of 2.17 , which we present in Table 6 .
We interpret the odds $\alpha_{k l}$ as follows: The frequency of a positive response to the FSE item is approximately 8.76 times greater when the response to the FSL item is positive (as opposed to negative) when the respondent's response to the FSC item and his/her sum score is held constant.

The estimates for Model 3 will be the same for men and women, subject to rounding error. They will differ for Model 4, of course, because of the specification that allows the item dependencies to differ by sex. Moving to the same set of expected frequencies for Model 4, for example, we get odds of 1.69 for women, which is our estimate of the parameter, $\alpha_{k l}$, and the log odds which we present in Table 6 . The odds for men are 18.86. Dividing these odds by the odds for women (1.69) yields 11.6, an estimate of the parameter $\alpha_{k l m}$. We interpret this parameter as follows: the conditional dependency between FSA and FSL is approximately 11 times greater for men than for women. The log of 11.6 is 2.45 , which we present in Table 6.

Finding a solution for the parameters $\alpha_{j k}$ and $\alpha_{j k m}$ is more complicated. Instead of estimating $\alpha_{j k}$, we can estimate the square of $\alpha_{j k}$, for example, dividing the product of the expected frequencies in cells 15 and 10 by the product of expected frequencies in cells 14 and 11 . We solve for $\alpha_{j k m}$ by taking the positive square root of this result. Having found separate estimates of $\alpha_{j k}$ for men and women, we can estimate $\alpha_{j k m}$ by dividing the estimate for men by the estimate for women. Once the parameters $\alpha_{j k}$ and $\alpha_{k l}$ in Model 3 and the parameters $\alpha_{j k}$, $\alpha_{j k m}, \alpha_{k l}$, and $\alpha_{k l m}$ in Model 4 are found, we can easily solve for the item difficulty parameters $\alpha_{i}$ and $\alpha_{l}$.

\section{DISCUSSION}

From the perspective of the Reiss scale, the good news in our results is that the items we examined have specific objectivity with respect to the respondent's sex, since we are unable to reject the null hypothesis that the itemdifficulty parameters are the same for men and women. 
Clearly, additional research on the remaining Reiss items, stratified by sex, is needed to see whether the assumption of specific objectivity holds for them as well. Also needed is research that uses other stratifying variables related to premarital sexual permissiveness, such as age, education, and religiosity, to probe this assumption further. Still, our results are powerful because of the strong relation between gender and premarital sexual permissiveness.

The bad news in our results, on the other hand, is the violation of local independence in the case of the FSA, FSL, and FSE items. These violations raise two issues: How does one interpret these results, and what are the implications for the measurement of premarital sexual permissiveness? With respect to interpretation, notice that the item dependencies occur between items adjacent in the interview schedule. This particular pattern of item dependencies raises the possibility of either: (1) a first-order autoregressive process in which the response to the FSA item affects the response to the FSL item, which, in turn, affects the response to the FSE item, or (2) a first-order moving average process in which the FSL and FSE items share the error terms of the preceding items (FSA and FSL, respectively). Although the interpretation of an autoregressive process is more attractive substantively, the interpretation of a moving average process is more consistent with the data. The former implies a diminished item dependency between the FSA and FSE items, whereas the latter does not. Because of the limited power of the analysis, however, it is difficult to distinguish between these two processes empirically. Finally, whatever choice we make in resolving the difference between these two interpretations, we still face the problem of accounting for the fact that the item dependencies are greater for men than for women. (Note that the four items we analyze concern the premarital sexual behavior of women. Possibly the item dependencies would be stronger for women in the case of items in which the target figure is a man. Naturally, this is a problem for future research).

Rather than concern ourselves too much with the interpretation of these complexities, however, we might heed Duncan's (1984b, p. 226) advice concerning results like ours to redesign the instrument in order to obtain a set of items that meet the criteria of the simple Rasch model. In this regard, note that both item dependencies involve the FSL item, full sexual relations for a woman when she is in love with her sexual partner. On the one hand, this result suggests that the word love is too close to both affection and engagement to sustain the assumption of local independence. Possibly the Reiss scale could be improved by eliminating this condition of sexual behavior from the Reiss scale. On the other hand, the 4 items that we analyze are the last of a set of 24 items that constitute the Reiss scale. If the item dependencies represent a response set, as we have suggested above, it is possible that this response set develops only after the repetition of essentially the same question of the approval of sexual behavior across the variations in the target figure's gender, the relation between the target figure's gender, the rela- tion between the target figure and his/her sexual partner, and the sexual behavior. In the first instance, we would find violations of local independence for all the love items in the scale and would recommend dropping these items from the scale. In the second instance, we would find the item dependencies only among the items located at the end of the scale and would recommend redesigning the interview schedule in order to reduce this response set.

\section{SUMMARY}

This paper shows how to use the SPSS log-linear subroutine to estimate the parameters and test the assumptions of the Rasch model, which is particularly suited to the analysis of items developed as a Guttman (or Guttmanlike) scale. Although the expression of the Rasch model as a log-linear model is well established in the statistical literature (Clogg, 1988; Duncan 1984b; Kelderman, 1984), in this paper we have attempted to make this connection more accessible by applying these methods in an analysis of items from the Reiss Premarital Sexual Permissiveness Scale, possibly the best candidate among the attitude measures in social psychology to meet the assumptions of the Rasch model. The key feature of the use of the log-linear subroutine in SPSS is to convert the values of the putative scale into a set of dummy variables and specify these dummy variables as covariates in the loglinear model (leaving out the dummy variable that corresponds to the reference category of the scale). The coefficients associated with these covariates are "nuisance parameters" and are ignored when one is interpreting the results of the analysis.

We began with the basic Rasch model (Model 1), which, in addition to the nuisance parameters, contains just main or marginal effects of the item variables. We then showed how to test specific violations of the Rasch model by comparing the basic model with less restrictive models. These less restrictive models fall into two categories: those that violate the assumption of local independence by allowing for conditional dependence between specific pairs of items, and those that violate the assumption of specific objectivity by allowing the item difficulty parameters to differ by the values of one or more control variables (the respondent's sex in the case of our analysis). We estimated these additional parameters by specifying interactions either between the items or between the items and the control variables. In our analysis, we found that only the first class of restrictive models provided a significant improvement in fit over the basic Rasch model. Although our discussion is limited to these models, the general comments readily extend to the second class of models. In describing the results, we have shown how to use the expected frequencies to calculate the parameter estimates these models generate in the SPSS output. Finally, we have concluded by discussing the implications of our results for the Reiss scale-in particular, how the results from this type of analysis can be used in the construction and modification of Guttman scales. 


\section{REFERENCES}

ANDERSEN, E. B. (1973). A goodness of fit test for the Rasch model. Psychometrika, 38, 123-140.

ANDERSEN, E. B. (1982). Latent trait models and ability parameters estimation. Applied Psychological Measurement, 4, 445-461.

ANDERSEN, E. B. (1988). Comparison of latent structure models. In R. Langeheine \& J. Rost (Eds.), Latent trait and latent class models (pp. 207-229). New York: Plenum.

ANDRICH, D. (1978). Relationship between Thurstone and Rasch approaches to item scaling. Applied Psychological Measurement, 3, $449-460$.

ANDRICH, D. (1982). Using latent trait measurement models to analyze attitudinal data: A synthesis of viewpoints. In D. Spearit (Ed.), The improvement of measurement in education and psychology: Contributions of latent trait theories (pp. 89-126). Hawthorn, Victoria, Australia: Australian Council for Educational Research.

ANDRICH, D. (1985). An elaboration of Guttman scaling with Rasch models for measurement. In N. B. Tuma (Ed.), Sociological methodology 1985 (pp. 33-80). London: Jossey-Bass.

ANDRICH, D. (1988). Rasch models for measurement. Beverly Hills, CA: Sage.

BAKer, R. J., \& Nelder, J. A. (1978). GLIM-manual, Release 3. Oxford: Royal Statistical Society.

ClogG, C. C. (1988). Latent class models for measuring. In R. Langeheine \& J. Rost (Eds.), Latent trait and latent class models (pp. 173205). New York: Plenum.

Clogg, C. C., \& Eliason, S. R. (1988). Some common problems in log-linear analysis. In J. S. Long (Ed.), Common problems/proper solutions: Avoiding error in quantitative research (pp. 226-257). Beverly Hills, CA: Sage.

Cressie, N., \& Holland, P. W. (1983). Characterizing the manifest probabilities of latent trait models. Psychometrika, 48, 129-141.

Delamater, J. (1986). Gender differences in sexual scenarios. In K. Kelley (Ed.), Females, males and sexuality: Theories and research (pp. 191-215). Albany, NY: SUNY Press.

Delamater, J., \& MacCorquodale, P. (1979). Premarital sexuality: Attitudes, relationships, behavior. Madison, WI: University of Wisconsin Press.

Duncan, O. D. (1984a). Notes on social measurement: Historical \& critical. New York: Russell Sage Foundation.

DunCAN, O. D. (1984b). Rasch measurement: Further examples and discussion. In C. F. Turner \& E. Martin (Eds.), Surveying subjective phenomena (Vol. 2, pp. 367-403). New York: Russell Sage Foundation.

Duncan, O. D. (1987). Are Likert scales unidimensional? Social Science Research, 16, 245-259.

Duncan, O. D., \& Stenbeck, M. (1988). Panels and cohorts: Design and model in the study of voting turnout. In C. C. Clogg (Ed.), Sociological methodology 1988 (pp. 1-35). London: Jossey-Bass.

FisCHER, G. H. (1974). Einfürung in die Theorie psychologischer Tests. [Introduction to psychological test theory]. Bern: Huber.

GoldsteIn, H. (1980). Dimensionality, bias, independence and measurement problems in latent trait test score test. British Journal of Mathematical \& Statistical Psychology, 33, 234-246.

GuSTAFFSON, J. E. (1979). PML: A computer program for conditional estimation and testing of the Rasch-model for dichotomous items. (Reports from the Institute of Education, No. 85). Gothenburg, Sweden: University of Gothenburg.
Haberman, S. J. (1978). Analysis of qualitative data (Vol. 1). New York: Academic Press.

Haberman, S.J. (1979). Analysis of qualitative data (Vol. 2). New York: Academic Press.

Hulin, C., Drasgow, F., \& Parsons, C. K. (1983). hem response theory: Application to psychological measurement. Homewood, IL: Dow Jones-Irwin.

Kelderman, H. (1984). Log-linear Rasch model tests. Psychometrika, 49, 223-245.

KEMPF, W. (1974). Dynamische Modelle zur Messung sozialer Vertaloungsdispositionen [Dynamic models for attitude measurement]. In W. Kempf (Ed.), Probabilistische Modelle in der Sozialpsychologie [Probabilistic models in social psychology] (pp. 13-55). Bern: Hans Huber.

LANGEHEINE, R., \& Rost, J. (1988). Introduction and overview. In R. Langeheine \& J. Rost (Eds.), Latent trait and latent class models (pp. 1-7). New York: Plenum.

MellenberG, G. J., \& ViJn, P. (1981). The Rasch model as a loglinear model. Applied Psychological Measurement, 5, 369-376.

MoKKeN, R. J. (1971). A theory and procedure of scale analysis. The Hague: Mouton.

RAsCH, G. (1960). Probabilistic models for some intelligence and attainment tests. Copenhagen: Danmark paedagogiske.

REISS, I. L. (1967). The social context of premarital sexual permissiveness. New York: Holt, Rinehart \& Winston.

SPSS user's guide. (1988). Chicago: SPSS Inc

TuUR, T. (1982). A connection between Rasch's item analysis model and a multiplicative Poisson model. Scandinavian Joumal of Statistics, 9, 23-30.

WOOD, R. L., WINGERSKY, M. S., \& LORD, F. M. (1976). LOGIST: A computer program for estimating ability and item characteristic curve parameters (Research Memorandum 76-6). Princeton, NJ: Educational Testing Service.

Wright, B. D., \&tone, M. H. (1979). Best test design: Rasch measurement. Chicago: Mesa Press.

\section{NOTES}

1. Cressie and Holland (1983) show how the Rasch model can be specified as a log-linear model with and without conditioning on the total score.

2. Note that although attitudes may differ between blacks and whites, this does not necessarily mean that the structure of the scale differs between the two groups. However, because this possibility exists and because there were too few blacks $(N=163)$ to investigate this, we decided to eliminate black respondents from the analysis. Eliminating respondents with missing responses on one or more of the four items reduced the effective sample size to 1,299 .

3. Despite our use of contrasts, the SPSS estimates of the main effects, FSL by FSA and FSE by FSL, average the estimates across men and women, whereas in Formula 6 we choose to set these effects equal to the estimates for women. To retain consistency with Formula 6 and Table 1, we present the latter estimates rather than the ones given in the SPSS output. On the other hand, all other parameter estimates for Model 4 agree with those provided by the SPSS output.

(Manuscript received February 21, 1992; revision accepted for publication March 23, 1993.) 